\title{
Advances in circulating tumor cells (ACTC): from basic research to clinical practice
}

\author{
Evi S Lianidou ${ }^{1 *}$, Dimitris Mavroudis ${ }^{2}$ and Klaus Pantel ${ }^{3}$
}

\begin{abstract}
The first 'Advances in Circulating Tumor Cells (ACTC): from Basic Research to Clinical Practice' meeting was held in Athens, Greece, September 26-29, 2012 (abstracts, presentations and a more detailed meeting report are freely available online: http://www.actc2012.org). We summarize in this report most major findings presented and the main conclusions derived during the expert panel sessions.
\end{abstract}

\section{Introduction}

Focused on state of the art research and on how to translate basic research on circulating tumor cells (CTCs) into clinical practice, the Advances in Circulating Tumor Cells (ACTC) meeting brought together 236 participants from 25 countries. Particular emphasis was given to understanding the biology of metastasis and molecular characterization of CTCs and to the relationship of CTCs with cancer stem cells. The designs and results of the first clinical trials to determine CTC clinical utility were discussed together with novel, state of the art technology platforms for CTC imaging, detection, quantification and molecular characterization. Standardization and quality control of CTC technologies and the need of comparison studies between different methodologies for CTC isolation and detection were intensely discussed. In the opening plenary lecture, Klaus Pantel (UKE, Hamburg, Germany) pointed out that early changes in CTC counts might indicate success or failure of a particular therapy given to an individual patient and that molecular analysis of CTCs can serve as a 'liquid biopsy' to reveal the specific characteristics of metastatic cancer cells. This information can be further used as companion diagnostics to improve the selection of appropriate therapies and obtain insights into therapy-induced selection of cancer cells.

\footnotetext{
* Correspondence: lianidou@chem.uoa.gr

'Department of Chemistry, University of Athens, 15771, Athens, Greece Full list of author information is available at the end of the article
}

\section{Recent advances in the biology of metastasis and cancer stem cells}

Jean Paul Thiery (BIOPOLIS, Singapore) pointed out that epithelial-mesenchymal transition (EMT) is likely to operate during the early stages of carcinoma invasion and that the mesenchymal-like state of carcinoma cells may confer stemness, protection from cell death, escape from immune response and, most importantly, resistance to conventional and targeted therapies. Cristoph Klein (University of Regensburg, Germany) emphasized the importance of the genetic disparity between primary tumors, disseminated tumor cells, and manifest metastases. Yibin Kang (Princeton University, USA) pointed out that vascular cell adhesion molecule 1 (VCAM1) is essential for the conversion from dormant bone micrometastases to macrometastasis and is a promising therapeutic target for inhibiting the aggressive conversion from dormancy to overt bone metastasis in breast cancer. As detailed by Julio Aguirre-Ghiso (Mt Sinai School of Medicine, USA), self-renewal and quiescence signals inhibit metastasis by inducing dormancy. The search for pharmacological tools and genes that could mimic a 'restrictive' microenvironment for disseminating tumor cells (DTCs) led to the identification of retinoic acid and the retinoic acid-inducible orphan nuclear receptor NR2F1. Danny Welch (Kansas University Cancer Center, USA) discussed mechanisms by which KISS1 may induce dormancy in DTCs at secondary sites and strategies whereby KISS1 could be exploited clinically to treat metastases. Andreas Trumpp (DKFZ, Heidelberg, Germany) has shown that transplantation of primary patient bloodderived CTCs induced metastatic growth in bones and liver, demonstrating the presence of metastasis-initiating cells. His data provide a molecular basis for the design of diagnostic tools to detect metastasis-initiating cells and for developing rational approaches to target metastasis in breast cancer. Hasan Korkaya (University of Michigan, USA) focused on the elucidation of molecular mechanisms mediating resistance to trastuzumab. By knocking down PTEN expression in HER2 overexpressing breast cancer cell lines he demonstrated that development of trastuzumab 
resistance is mediated by activation of an interleukin- 6 inflammatory feedback loop.

Michael Clarke (Stanford University, USA) announced that his group identified two distinct normal mammary stem cell populations that had similar engraftment ability in vivo, demonstrating that there are likely two physiological stem cell states. Single cell analysis of estrogen receptor-negative tumors revealed a unique cancer stem cell population that differs from the one found in estrogen receptor-positive tumors. Max Wicha's (University of Michigan Comprehensive Cancer Center, USA) group characterized breast cancer stem cells (BCSCs) across molecular subtypes of breast cancer and found that CD44+/CD24- and aldehyde dehydrogenase identify different cell populations with varying degrees of overlap. CD44+/CD24- identifies a BCSC population that expresses markers of EMT while, in contrast, aldehyde dehydrogenase marks a more epithelial self-renewing cancer stem cell population. Wicha proposed a model in which metastasis is dependent on BCSC state transitions regulated by the tumor microenvironment and microRNAs.

\section{Clinical utility of circulating tumor cells}

Tanjia Fehm (University of Dusseldorf, Germany) has shown that hematogenous and lymphatic tumor cell dissemination is detected even in patients diagnosed with ductal carcinoma in situ of the breast. Michail Ignatiadis (Jules Bordet Institute, Belgium) pointed out that although selection of an aggressive HER2+ tumor clone during progression from early to metastatic disease in HER2- breast cancer cannot be excluded, limitations of detection technologies seem to account for many discordant cases between primary tumor versus metastasis/CTCs. Wolfgang Janni (University of Ulm, Germany) showed that the SUCCESS trial confirmed independent prognostic relevance of CTCs in 2,026 patients with primary breast cancer. CTCs were detected in $21.5 \%$ of patients before the start of adjuvant chemotherapy and their presence predicted poor disease-free survival, distant disease-free survival and overall survival. According to Bjorn Naume (Oslo University Hospital, Norway) the presence of DTCs after neoadjuvant chemotherapy indicated high risk for relapse and death, irrespective of the DTC status before treatment, supporting the potential use of DTC analysis as a monitoring tool during follow-up for selection of patients for secondary treatment intervention within clinical trials. In the first line metastatic setting, Jean Yves Pierga (Curie Institute, France) discussed the results from the IC 2006-04 study, according to which elevated CTCs before $\mathrm{C} 2$ was an early predictive marker of poor progression-free survival and overall survival, and could be used to monitor treatment benefit. Dimitris Mavrudis (University of Crete, Greece) indicated that persistent detection of CK-19 mRNA-positive CTCs during the first 5 years of follow-up is associated with an increased risk of late relapse and death in patients with operable breast cancer and indicates the presence of chemo- and hormonotherapy-resistant residual disease. The administration of trastuzumab could eliminate chemotherapyresistant CK19 mRNA-positive CTCs and reduce the risk of disease recurrence. A novel marker, PLASTIN-3 (PLS3), that is specific for CTCs undergoing EMT and is associated with colorectal cancer prognosis was presented by Koshi Mimori (Kyushu University, Japan). Caroline Dive (Paterson Institute for Cancer Research, UK) discussed the clinical significance and molecular characteristics of circulating tumor microemboli detected in patients with small-cell lung cancer undergoing standard treatment.

\section{State of the art technologies for the isolation, detection and characterization of circulating tumor cells}

Richard Cote (Miller School of Medicine, Miami, USA) described the potential of a portable novel parylene-based portable microfilter device for CTC capture, enumeration, and characterization. Leon Terstappen (University of Twente, Netherlands) showed that EpCAM+, cytokeratin+, CD45- nucleated CTCs are present in all patients with metastatic breast, prostate, and colorectal cancer and their frequency is proportional to survival. Catherine AlixPanabières (Montpellier, France) described the potential of the EPISPOT assay for CTC analysis that can detect proteins secreted/released/shed from viable single epithelial cancer cells. Peter Kuhn (Scripps, San Diego, USA) presented a high definition imaging system for CTCs that can be used as a real-time window into cancer evolution in individual patients. As detailed by Sabine Kasimir-Bauer (University of Essen, Germany), a subset of primary breast cancer patients shows EMT and stem cell characteristics that currently used detection methods for CTCs are not efficient at identifying. Marija Balic (University of Graz, Austria) focused on putative BCSC markers in CTCs and evaluated the feasibility of triple and quadruple labeling of breast cancer cells by employing a novel DyLight Technology. Evi Lianidou (University of Athens, Greece) demonstrated the power and potential of multiplex quantitative RT-PCR for the molecular characterization of CTCs. Massimo Cristofanilli (Jefferson Breast Care Center, Philadelphia, USA) showed that patients with HER2+ metastatic breast cancer have EMT-CTCs. Concerning melanoma, Dave Hoon (John Wayne Cancer Institute, Santa Monica, USA) pointed out that CTC status is a prognostic factor for recurrence-free survival, distant metastasis disease-free survival, and melanoma-specific survival after complete lymphadenectomy in patients with sentinel lymph node metastasis and that multi-marker quantitative RT-PCR analysis may be useful in discriminating patients who may benefit from aggressive adjuvant therapy or stratifying patients for adjuvant clinical trials. Michael Mindrinos (Stanford Genome Technology Center, US) presented novel technology that will be vital for CTC analysis in the near 
future based on next-generation sequencing approaches. George Calin (MD Anderson Cancer Center, USA) discussed the novel discoveries and future challenges concerning the role that microRNAs play in metastasis.

\section{Expert Panel and special topics sessions}

During two expert panels, future challenges of CTC research in personalized medicine and the next steps in CTC technology development were discussed, while in two special sessions on the 'Potential Applications of Circulating Tumor Cells in Clinical Drug Development' and on the 'State of the art technological platforms for CTC isolation, enumeration and molecular characterization' renowned speakers in the CTC field exchanged ideas. All these sessions generated a lot of questions and very intense and fruitful discussions on the potential and limitations of current CTC technology, especially concerning the requirements for establishing CTCs as biomarkers in the clinical setting.

\section{Conclusion and future perspectives}

The 'liquid biopsy' approach based on the monitoring, enumeration and molecular characterization of CTCs is rapidly becoming a valuable tool in translational medicine, especially for the prediction of therapeutic efficacy in cancer patients. Molecular characterization of CTCs will contribute to a better understanding of the biology of metastasis, may improve stratification and will help towards the identification of novel therapeutic targets and understanding resistance to therapies. Single cell analyses might be useful for developing new strategies to treat cancer by identifying the expression of therapeutic targets in each of the cancer cell subpopulations that make up a particular tumor. However, CTC isolation and molecular characterization technologies should first be validated in respect to sensitivity and specificity and robustness and cross-validated by experienced labs before application in the clinical setting.

Abbreviations

ACTC: Advances in circulating tumor cells; BCSC: Breast cancer stem cell; CTC: Circulating tumor cell; DTC: Disseminating tumor cell; EMT: Epithelialmesenchymal transition; RT-PCR: Reverse transcriptase-polymerase chain reaction.

\section{Competing interests}

The authors declare that they have no competing interests.

\section{Acknowledgements}

We would like to thank all participants for the excellent scientific contributions and all sponsors for their generous support. We would like also to thank the members of the International Scientific Committee for their contribution to the evaluation of all abstracts and for the selection of the abstracts for Oral presentations, Posters and Travel Awards. Especially we would like to thank the Hellenic Society of Medical Oncology for its generous support and for sponsoring all ACTC travel grants and awards. We apologize to all those participants whose work has not been mentioned owing to space constraints. A complete listing of all participants, speakers, oral presentations, posters and abstracts are available at http://www.actc2012.org.

\section{Author details}

'Department of Chemistry, University of Athens, 15771, Athens, Greece.

${ }^{2}$ Department of Medical Oncology, University Hospital of Heraklion, School of Medicine, University of Crete, Crete 71110, Greece. ${ }^{3}$ Institute of Tumor Biology, University Medical Center Hamburg-Eppendorf, Martinistrasse 52, 20246, Hamburg, Germany.

Published: 05 Dec 2013

\section{$10.1186 /$ bcr3582}

Cite this article as: Lianidou et al:: Advances in circulating tumor cells (ACTC): from basic research to clinical practice. Breast Cancer Research 2013, 15:319 